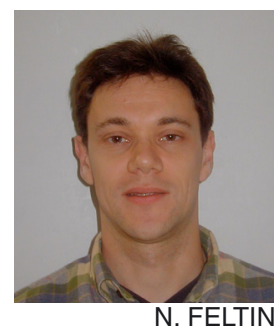

\title{
Trimet : fermeture du triangle métrologique quantique à un niveau d'incertitude relative de $10^{-6}$
}

\section{Trimet: closing the quantum metrological triangle at a relative uncertainty level of $10^{-6}$}

\author{
Nicolas FELTIN, Barthélémy STECK, Laurent DEVOILLE, Sami SASSINE, Boris CHENAUD, Wilfrid \\ POIRIER, Félicien SCHOPFER, Guillaume SPRENGLER, Sophie DJORDJEVIC, \\ Olivier SERON et François PIQUEMAL
}

Laboratoire national de métrologie et d'essais (LNE), 29 Avenue Roger Hennequin, 78197 Trappes Cedex, France, nicolas.feltin@lne.fr.

\section{Résumé}

Nous présentons les principaux résultats obtenus dans le cadre du projet ANR-TRIMET dont l'objectif était la fermeture du triangle métrologique quantique (TMQ) à un niveau d'incertitude relative de $10^{-6}$. L'expérience du TMQ consiste à réaliser une loi d'Ohm en utilisant les trois effets quantiques impliqués en métrologie électrique : l'effet Josephson (EJ), l'effet Hall quantique (EHQ) et l'effet tunnel à un électron (SET). Le but est de vérifier la cohérence des constantes phénoménologiques $K_{\mathrm{J}}, R_{\mathrm{K}}, Q_{\mathrm{X}}$, associées respectivement à ces trois effets et théoriquement exprimées en fonctions des deux constantes fondamentales, $h$ et $e$ (constante de Planck et charge élémentaire). Cette expérience est une contribution importante à une redéfinition du Système international d'unités (SI). Nous montrons aussi que la fermeture du TMQ permettra la mise en œuvre d'une nouvelle détermination de la charge élémentaire, $e$.

MOTS CLÉS : MÉTROLOGIE ÉLECTRIQUE QUANTIQUE, CONSTANTE FONDAMENTALE, EFFET TUNNEL, DISPOSITIF MONOÉLECTRONIQUE.

\section{Abstract}

We present our experimental set-up and discuss the results obtained within the framework of the ANR-TRIMET project which aim was the closure of the quantum metrological triangle (QMT) at a relative uncertainty level of 1 part in $10^{6}$. This experiment consists in achieving Ohm's law with the three effects used and investigated in quantum electrical metrology: Josephson effect (JE), quantum Hall effect $(Q H E)$ and single electron tunnelling effect (SET). The aim is to check the consistency of the phenomenological constants $K_{\mathrm{J}}, R_{\mathrm{K}}$ and $Q_{\mathrm{X}}$ associated with these effects and theoretically expressed with the fundamental constants $e$ and h (elementary charge and Planck constant, respectively).
Such an experiment is a contribution for a new definition of the International System of Units (SI). Also, the obtained results are a first step towards a determination of $e$.

KEY WORDS: QUANTUM ELECTRICAL METROLOGY, FUNDAMENTAL CONSTANT, TUNNELING EFFECT, SINGLE ELECTRON DEVICE.

Sigles utilisés
$2 D E G$ :
2 Dimensional Electron Gas.
ANR : $\quad$ Agence National de la Recherche, France.
BIPM : Bureau international des poids et mesures.
CCC : $\quad$ Comparateur Cryogénique de Courants.
CGPM : Conférence générale des poids et mesures.
CIPM : Comité international des poids et mesures.
CNAM : Conservatoire national des arts et métiers.
CODATA : Committee on Data for Science and Technology.
DAC: Digital Analog Converter (converstisseur analogique- numérique).
EHQ : $\quad$ Effet Hall Quantique.
EJ : $\quad$ Effet Josephson.
JAVS : Josephson Array Voltage Standard.
LCM : $\quad$ Laboratoire commun de métrologie LNE-CNAM.
LNE : Laboratoire national de métrologie et d'essais, France.
LNM : laboratoire national de métrologie.
LPL : $\quad$ Laboratoire de Physique des Lasers, France. 


$\begin{array}{ll}\text { METAS : } & \text { Office fédéral de métrologie, Suisse. } \\ \text { NIST : } & \begin{array}{l}\text { National Institute of Standards and Technology, États- } \\ \text { Unis d'Amérique. }\end{array} \\ \text { NMIA : } & \text { National Metrology Institute of Australia. } \\ \text { NPL : } & \text { National Physical Laboratory, Royaume-Uni. } \\ \text { PTB : } & \text { Physikalisch-Technische Bundesanstalt, Allemagne. } \\ \text { SET : } & \text { Single Electron Tunneling. } \\ \text { SI : } & \text { Système international d'unités } \\ \text { SQUID : } & \text { Superconducting Quantum Interference Device. } \\ \text { TMQ : } & \text { Triangle Métrologique Quantique. }\end{array}$

\section{Introduction}

L'ensemble des laboratoires nationaux de métrologie (LNM) est impliqué dans le projet historique de révision du Système international d'unités (SI), dont l'objectif est la redéfinition de certaines unités de base à partir de valeurs fixées d'un nombre restreint de constantes fondamentales. Ces «constantes de la nature» présentent des avantages considérables et conféreraient au futur SI une base solide. En effet, elles décrivent les propriétés universelles de la matière et de ses interactions, et sont indépendantes de tout référentiel considéré ainsi que de sa position dans l'espace et le temps.

Cette profonde modification du SI est une conséquence logique de la dématérialisation, entreprise depuis trois décennies, des étalons fondamentaux représentant certaines unités. Ces travaux ont été motivés par l'introduction de la physique fondamentale dans le domaine de la métrologie (physique des lasers, atomes froids, effet Josephson, effet Hall quantique...) et facilités par la mise en œuvre de nouvelles technologies (laser femtoseconde, interférométrie atomique, peigne de fréquences, horloges optiques, SQUID et comparateur cryogénique de courants...) [1-3]. Cette révolution a débuté avec l'unité de temps, la seconde, aujourd'hui reliée à l'énergie de transition entre deux niveaux hyperfins de l'atome de césium, puis, avec la fixation de la vitesse de la lumière dans le vide, $c_{0}$, permettant une redéfinition du mètre à partir de la seconde [4].

Le réseau français de métrologie, piloté par le LNE, a accompagné ces récents progrès et consacré de nombreux efforts afin d'améliorer le système d'unités en participant à la majorité des expériences clés qui contribueront à l'adoption de ce futur SI. L'enjeu est de taille et le calendrier du comité international des poids et mesures (CIPM) prévoit que ce dernier jugera d'une possible redéfinition du kilogramme, de l'ampère, du kelvin et de la mole en utilisant des valeurs fixées de certaines constantes de la physique lors de la $24^{\mathrm{e}}$ Conférence générale des poids et mesures en 2011 [5].

Ainsi, à l'instar du NIST, du NPL et du METAS, le LNE a entrepris de mettre en œuvre l'expérience de la balance du watt dont l'objectif est de dématérialiser le prototype international du kilogramme en reliant l'unité de masse à la constante de Planck, $h$ [6]. Cette expérience est fondée sur l'équivalence entre puissance électrique et puissance mécanique, et établit un lien entre unités électrique et mécanique au travers de $h$.

Le kelvin, quant à lui, est défini dans le SI à partir du point triple de l'eau pure qui constitue le point fixe fondamental de l'échelle thermodynamique absolue. Cependant, une nouvelle définition de l'unité de température thermodynamique à partir de la constante de Bolzmann, $k$, pourrait présenter de nombreux avantages. Or, la valeur actuelle de cette constante a une incertitude qui est jugée trop grande. Le LCM/CNAM s'est engagé dans la détermination de la constante de Boltzmann en espérant atteindre de meilleurs niveaux d'incertitudes grâce à deux méthodes expérimentales : une méthode spectroscopique (en collaboration avec le LPL) [7] et une méthode acoustique (développement d'un résonateur acoustique sphérique contenant un gaz pur maintenu à la température du point triple de l'eau) [8].

Dans le domaine de la métrologie électrique, le LNE est l'un des rares LNM a posséder une activité sur les trois étalons quantiques électriques (intensité de courant, tension et résistance) [9]. Pour cela, il a été nécessaire, d'une part, de poursuivre les efforts dans la maîtrise de l'effet Josephson et de l'effet Hall quantique pour les étalons primaires de tension et de résistance, et d'autre part de développer un étalon de courant fondé sur un troisième effet quantique : l'effet tunnel mono-électronique (voir § 2) [10]. Cette stratégie permet au LNE d'avoir une position incontournable dans les débats actuels sur la définition d'un ampère «quantique » et ainsi de mettre en œuvre l'expérience du triangle métrologique quantique (TMQ) [11-13]. L'objectif de cette expérience est d'approfondir nos connaissances sur les trois effets quantiques afin de pouvoir définir, à terme, l'ensemble des unités électriques à partir de $h$ et $e$, charge élémentaire.

Nous proposons ici de donner l'ensemble des résultats obtenus dans le cadre du projet ANR-TRIMET. Le financement de ce projet a permis au LNE de mettre en œuvre, pour la première fois, l'expérience du triangle métrologique. Comme nous le verrons dans la seconde partie de l'article, cette expérience revient à vérifier une loi d'ohm « quantique ». L'expérience du TMQ aura un impact majeur sur les discussions autour du futur SI, si l'incertitude relative sur la vérification de cette loi d'ohm « quantique » est réduite à $10^{-8}$. Mais une étape intermédiaire a été définie pour ce projet à $10^{-6}$. Il s'agissait ainsi de valider les options retenues pour le développement du montage expérimental et de vérifier la faisabilité de l'expérience.

\section{Contexte}

\subsection{La métrologie électrique quantique}

Avant 1990, les unités de tension électrique et de résistance électrique du SI étaient maintenues dans chaque laboratoire national par l'intermédiaire d'étalons matériels. La détermination de la valeur absolue de ces étalons s'effectuait par la mise en place d'expériences complexes donc rarement effectuées. 


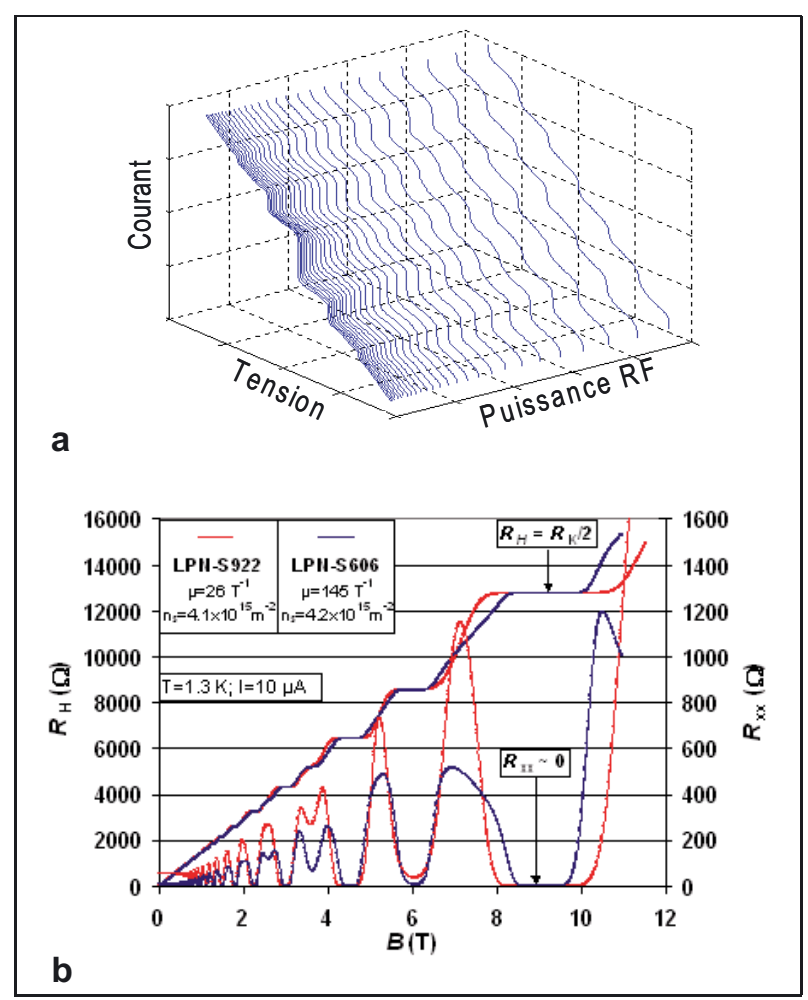

Fig. 1. - (a) Série de caractéristiques courant-tension d'une jonction Josephson en fonction de la puissance radiofréquence appliquée faisant apparaître des «marches de tension» à des multiples entiers de la valeur $K_{\mathrm{J}}^{-1} f_{\mathrm{J}}$ («marches de Shapiro »). (b) Caractéristique résistance-champ magnétique d'un échantillon de Hall : la résistance de Hall $R_{\mathrm{H}}$ est parfaitement quantifiée (plateaux) et vaut $R_{\mathrm{K}} / i$, où $i$ est l'indice du plateau considéré.

Les découvertes au cours des années 1970 et 1980 des effets Josephson (EJ) et Hall quantique (EHQ) ont permis d'élaborer des étalons électriques de représentation du volt et de l'ohm mettant en œuvre des constantes fondamentales ( $h$, constante de Planck, et $e$, charge élémentaire) avec de faibles incertitudes (gain de 4 à 5 ordres de grandeurs par rapport aux étalons traditionnels). En outre, les premières études ont prouvé une grande stabilité dans le temps de ces nouveaux étalons et une grande reproductibilité ; d'un point de vue fondamental, ils revêtaient un caractère universel. Cette évolution profonde a permis l'avènement de la métrologie électrique quantique.

L'effet Josephson (EJ) correspond au passage de paires de Cooper à travers une jonction tunnel au sein d'un circuit supraconducteur à basse température, lorsque celle-ci est irradiée par des photons micro-ondes. Le courant de paires va alors se synchroniser avec la fréquence du rayonnement, $f_{\mathrm{J}}$, (et ses harmoniques) et une tension continue apparaît aux bornes de la jonction. Ceci se traduit par l'apparition de «marches de tension » à des multiples de la valeur quantifiée $K_{\mathrm{J}}^{-1} \cdot f_{\mathrm{J}}$ dans les caractéristiques courant-tension, appelées «marches de Shapiro» (Fig. 1a). La tension Josephson peut alors s'écrire avec la relation (1).

$$
U_{\mathrm{J}}=n \cdot K_{\mathrm{J}}^{-1} \cdot f_{\mathrm{J}},
$$

où $n$ est un nombre entier et $K_{\mathrm{J}}$ la constante Josephson [14].

L'effet Hall quantique (EHQ) est un phénomène de transport électronique qui apparaît dans un gaz d'électrons bidimensionnel (réalisé par exemple à l'interface d'une hétérostructure $\mathrm{AlGaAs} / \mathrm{GaAs}$ ). Cet effet, observable à basse température $(4,2 \mathrm{~K})$ et à densité de flux magnétique intense (typiquement supérieur à $10 \mathrm{~T}$ ) est caractérisé par la quantification de la résistance de Hall (apparition de plateaux de résistance en Fig. 1b) comme l'indique la relation (2).

$$
R_{\mathrm{H}}(i)=\frac{R_{\mathrm{K}}}{i}
$$

où $i$ est un entier et $R_{\mathrm{K}}$ la constante de von Klitzing [15].

Les deux effets, EJ et EHQ, impliquent donc directement deux constantes phénoménologiques, $K_{\mathrm{J}}$ et $R_{\mathrm{K}}$, qui sont théoriquement reliées aux constantes fondamentales $e$ et $h$ par les expressions (3) et (4) :

$$
\begin{aligned}
K_{\mathrm{J}} & \equiv \frac{2 e}{h} \\
R_{\mathrm{K}} & \equiv \frac{h}{e^{2}} .
\end{aligned}
$$

En théorie, ces deux constantes représentent respectivement l'inverse du quantum de flux, $\phi_{o}$, et le quantum de résistance.

Depuis 1990, de nombreux tests d'universalité ont été effectués sur EJ et EHQ renforçant ainsi notre confiance dans les égalités (3) et (4) et sur le caractère universel de ces deux effets.

À l'instar des expériences de Clarke [16], de nombreux tests ont établi une limite comprise entre $2 \times 10^{-16}$ et quelques $10^{-19}[17,18]$ sur l'indépendance de la tension vis-à-vis de la fréquence du rayonnement $f_{\mathrm{J}}$ et ceci, quelle que soit la nature chimique de la jonction Josephson et quels que soient les paramètres expérimentaux.

Concernant l'EHQ, les tests d'universalité effectués démontrent l'indépendance du produit $R_{\mathrm{H}}(i) \cdot i$ sur un nombre important de dispositifs avec des incertitudes de l'ordre de quelques $10^{-10}$ [19-21]. Une nouvelle génération de tests est réalisée au LNE grâce à la mise au point d'un pont de Wheatstone «quantique » capable d'atteindre des incertitudes inférieures à $10^{-10}$. Des résultats préliminaires validant le montage expérimental ont montré un écart inférieur à $2 \times 10^{-12}$ entre quatre dispositifs barres de Hall avec une incertitude relative de $3,2 \times 10^{-11}[22,23]$. Des tests sont actuellement en cours avec des matériaux de natures différentes (2DEGs III-V GaAs/AlGaAs de propriétés différentes, 2DEG II-VI HgTe/HgCdTe,...). Enfin, le LNE a entrepris des études plus prospectives concernant l'étude de l'EHQ dans le graphène [24]. L'introduction d'une feuille de graphène dans le pont de Wheatstone et une comparaison de la quantification de la résistance de Hall au sein de ce matériau révolutionnaire avec une hétérostucture de 
semiconducteurs de type GaAs/AlGaAs constituerait un test ultime de l'universalité de l'effet Hall quantique. En effet, la physique du graphène est si exotique et différente de celle des semiconducteurs usuels, qu'une comparaison même à $10^{-8}$ près apporterait de précieuses informations. Des premiers résultats ont été récemment obtenus avec ces incertitudes [25, 26].

Cependant, malgré toutes ces études, nous ne pouvons pas définitivement conclure que les égalités (3) et (4) sont exactes, c'est-à-dire dénuées de tout facteur de correction. Bien sûr, les tests d'universalité nous indiquent que, si ces facteurs existent, ils ne sont liés ni à la nature des matériaux, ni à des paramètres environnementaux mais à des phénomènes plus fondamentaux.

Mais les incertitudes relatives actuelles associées aux valeurs mesurées de $R_{\mathrm{K}}\left(1 \times 10^{-7}\right)$ et $K_{\mathrm{J}}\left(4 \times 10^{-7}\right)$ en unités SI sont au minimum de deux ordres de grandeur plus grandes que celles annoncées plus haut. La valeur de $R_{\mathrm{K}}$ découle de déterminations directes de l'ohm via un étalon calculable de capacité de type Thompson-Lampard et des valeurs de $h / e^{2}$ déduites de mesures indirectes. $K_{\mathrm{J}}$ a été déduite, en partie, des mesures effectuées avec deux balances du watt (NIST et NPL), des expériences faites avec un électromètre (NMIA, PTB) ou avec la balance du volt (NPL, NIST) [9, 27].

Cet écart entre reproductibilité des phénomènes quantiques et incertitudes liées aux constantes mesurées dans le SI explique le fossé qui s'est peu à peu creusé entre la physique moderne et le SI actuel. Ainsi la métrologie électrique a pris ses distances, devenant pratiquement indépendante du SI, en adoptant en 1990 (avec la recommandation du CIPM), des valeurs conventionnelles pour les constantes de Josephson, $K_{\mathrm{J}-90}$, et von Klitzing $R_{\mathrm{K}-90}$. L'attribution de valeurs fixes à ces deux constantes permet aux chaînes d'étalonnage de bénéficier du caractère universel des deux effets quantiques associés [28, 29]. Cette solution permet d'assurer la cohérence des représentations du volt et de l'ohm malgré les limitations rencontrées pour la détermination de $R_{\mathrm{K}}$ et $K_{\mathrm{J}}$. Parallèlement, les métrologues affinent continuellement la détermination directe de ces constantes dans le SI.

\subsection{Les enjeux de l'expérience du triangle métrologique quantique}

L'expérience du triangle métrologique quantique (TMQ) apporte une contribution importante à la vérification des relations (3) et (4), étape nécessaire pour redéfinir les unités électriques à partir de $e$ et $h$.

Dans sa version la plus naturelle, cette expérience s'appuie sur l'application d'une loi d'Ohm schématisée en figure 2 et formulée selon la relation (5).

$U_{\mathrm{J}}=R_{\mathrm{H}} \cdot I_{\mathrm{SET}}$,

où $R_{\mathrm{H}}$ est la résistance de Hall, $U_{\mathrm{J}}$ la tension Josephson de référence et $I_{\mathrm{SET}}$ l'intensité du courant généré par un dispositif mono-électronique.

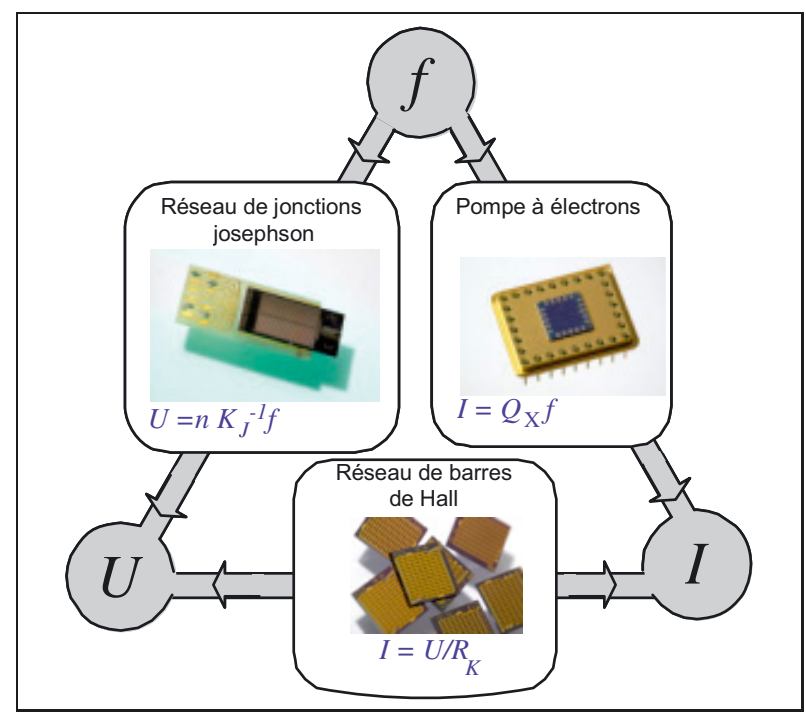

Fig. 2. - Principe de l'expérience du triangle métrologique.

D'après l'équation (5), la mise en œuvre du TMQ nécessite ainsi l'implication d'un troisième effet quantique, l'effet tunnel mono-électronique (SET), compatible avec l'EHQ et l'EJ, et associé à une relation phénoménologique supplémentaire (6).

$$
Q_{\mathrm{X}} \equiv e,
$$

où $Q_{\mathrm{X}}$ représente un estimateur de la charge de l'électron généré par un dispositif SET.

Au début des années 1980, les travaux de Fulton et Dolan [30] sur les dispositifs SET ont ouvert la voie à l'électronique à un électron. Ces dispositifs mésoscopiques fondés sur le principe du blocage de Coulomb sont capables de générer ou de contrôler le passage des électrons un à un à travers un circuit électrique [31]. Le phénomène de blocage de Coulomb apparaît lorsqu'un morceau de circuit, appelé îlot, est électriquement isolé du reste du circuit grâce à deux jonctions tunnel (Fig. 3a). Tant que l'énergie des électrons est inférieure à l'énergie de Coulomb, le flux d'électrons est bloqué et le courant est nul. Le système peut être débloqué de deux manières : soit en appliquant une tension de polarisation aux bornes du dispositif, soit en modifiant l'état de charge de l'îlot par l'intermédiaire d'une tension $V_{\mathrm{g}}$ appliquée à une électrode dite « de grille» (Fig. 3a).

Le dispositif le plus étudié dans les laboratoires de métrologie est la pompe à électron dont la version la plus simple est donnée en figure 3b. Ce dispositif SET métallique (Al) est constitué de trois jonctions tunnel $\left(\mathrm{Al}_{2} \mathrm{O}_{3}\right)$ formant deux îlots couplés à deux électrodes de grilles. Le transfert des électrons un par un à travers le système est réalisé de manière quasi-adiabatique avec une fréquence ajustable, $f_{\mathrm{SET}}$. Par conséquent, l'intensité du courant fournie par un tel dispositif s'écrit théoriquement avec la relation (7).

$$
I_{\text {pump }}=Q_{\mathrm{X}} \cdot f_{\mathrm{SET}} \text {. }
$$




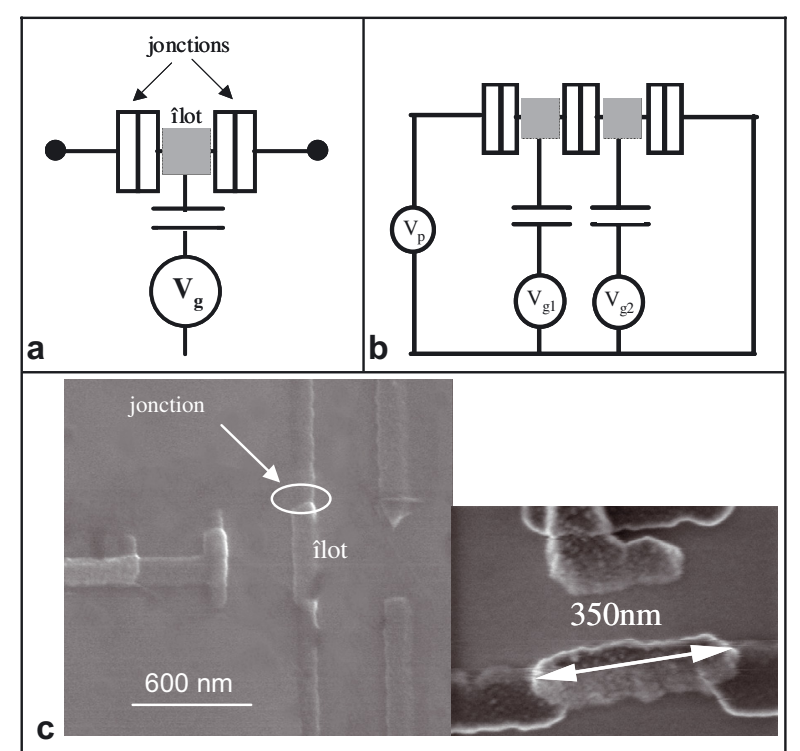

Fig. 3. - (a) Représentation schématique d'un îlot métallique dont l'état de charge est contrôlé par une électrode de grille; (b) représentation schématique d'une pompe à électron; (c) image d'un îlot et d'une électrode de grille réalisée avec microscope électronique à balayage. Les jonctions tunnel métal/isolant/métal ont été réalisées par microlithographie électronique par le LPN grâce à la méthode de la métallisation sous angles à travers un masque suspendu (à droite, un îlot de $350 \mathrm{~nm})$.

Les pompes à électron étudiées au LNE ont été fabriquées, pour certaines, au PTB et, pour d'autres, au LPN (Fig. 3c). Le fonctionnement d'une pompe ainsi que les principaux résultats obtenus sur ces dispositifs sont reportés dans les articles $[9,10,32,33]$. Le courant maximum atteint avec de tels dispositifs est d'environ $16 \mathrm{pA}$ $\left(f_{\mathrm{SET}}=100 \mathrm{MHz}\right)$.

Les propriétés métrologiques des pompes à électron sont étudiées au laboratoire. Nous savons que le passage quantifié de charges au travers de la pompe peut être perturbé à cause, principalement, de trois phénomènes : erreurs dues à l'augmentation de la fréquence de pompage, erreurs dues à la température des électrons dans la jonction et enfin transferts non contrôlés à cause d'un effet quantique appelé effet tunnel « concomitant» (cotunnelling). Deux résistances lithographiées en chrome sont ajoutées aux extrémités de la pompe à électron afin de minimiser cette dernière source d'erreur [34].

Pour la mise en pratique du TMQ, les courants générés par les pompes à électron sont trop faibles et doivent être amplifiés. L'un des points forts du laboratoire est la maîtrise d'un outil métrologique appelé «comparateur cryogénique de courants continus (CCC)» [35]. Il s'agit d'un transformateur de flux dont la particularité réside dans le fait que le gain d'amplification du courant est connu avec une incertitude très faible [36]. Par conséquent, l'égalité (5) devient (8).

$$
U_{\mathrm{J}}=R_{\mathrm{H}} \cdot N_{\mathrm{CCC}} \cdot I_{\text {pump }}
$$

où $N_{\mathrm{CCC}}$ est le gain du CCC.
Dans le domaine de la métrologie du volt, les progrès en microfabrication de ces dernières années ont permis de réaliser des réseaux de plusieurs milliers de jonctions Josephson. L'avantage de ces réseaux réside dans la possibilité de sélectionner sans ambiguïté les «marches » de tension dans un temps très court. L'architecture des réseaux programmables est fondée sur une répartition des jonctions Josephson en séquences binaires appelées segments [28]. Une mise en pratique du TMQ nécessite l'utilisation d'un tel réseau programmable.

En tenant compte des relations (1) et (2), (8) peut être réécrite sous la forme de l'équation (9).

$$
n_{\mathrm{J}} \cdot \frac{f_{\mathrm{J}}}{K_{\mathrm{J}}}=\frac{R_{\mathrm{K}}}{i} \cdot N_{\mathrm{CCC}} \cdot I_{\mathrm{pump}}
$$

Attention, $n_{\mathrm{J}}$ n'est pas ici le numéro de la «marche» mais représente le nombre de jonctions du réseau Josephson (JAVS) en considérant la tension mesurée sur la première "marche » et en appliquant la fréquence micro-onde, $f_{\mathrm{J}}$. $i$ est l'indice du plateau de Hall de l'étalon quantique de résistance.

(9) peut ainsi s'écrire :

$$
R_{\mathrm{K}} \cdot K_{\mathrm{J}} \cdot Q_{\mathrm{X}}=\frac{f_{\mathrm{J}}}{f_{\mathrm{SET}}} \cdot \frac{n_{\mathrm{J}} \cdot i}{N_{\mathrm{CCC}}} .
$$

Si on considère $\varepsilon_{K}$, $\varepsilon_{J}$ et $\varepsilon_{X}$, trois facteurs de correction correspondant aux trois constantes phénoménologiques, définis à partir des relations (11), (12) et (13) :

$$
\begin{aligned}
K_{\mathrm{J}} & =\frac{2 e}{h} \cdot\left(1+\varepsilon_{\mathrm{J}}\right) \\
R_{\mathrm{K}} & =\frac{h}{e^{2}} \cdot\left(1+\varepsilon_{\mathrm{K}}\right) \\
Q_{\mathrm{X}} & =e \cdot\left(1+\varepsilon_{\mathrm{X}}\right) .
\end{aligned}
$$

La relation (10), simplifiée au premier ordre, devient :

$$
1+\left(\varepsilon_{\mathrm{J}}+\varepsilon_{\mathrm{K}}+\varepsilon_{\mathrm{X}}\right)=\frac{1}{2} \cdot \frac{f_{\mathrm{J}}}{f_{\mathrm{SET}}} \cdot \frac{n_{\mathrm{J}} \cdot i}{N_{\mathrm{CCC}}}
$$

Il est à noter que toutes les variables situées à droite de l'égalité (14) sont connues avec des incertitudes extrêmement faibles et ces quantités sont ajustées de telle sorte que le produit vaille 1.

$$
\left(\varepsilon_{\mathrm{J}}+\varepsilon_{\mathrm{K}}+\varepsilon_{\mathrm{X}}\right) \text { est donc l'écart à l'égalité } 1=1 \text { ! }
$$

La réalisation expérimentale du triangle métrologique revient à mesurer l'égalité :

$$
1+\left(\varepsilon_{\mathrm{J}}+\varepsilon_{\mathrm{K}}+\varepsilon_{\mathrm{X}}\right) \pm \sigma_{\mathrm{TMQ}}=1,
$$

où $\sigma_{\mathrm{TMQ}}$ est l'écart type relatif associé à la mesure de $\left(\varepsilon_{\mathrm{J}}+\varepsilon_{\mathrm{K}}+\varepsilon_{\mathrm{X}}\right)$. Dans le cas où cette dernière variable est inférieure à $\sigma_{\mathrm{TMQ}}$, le triangle métrologique est dit «fermé » et aucune des corrections n'est significative 


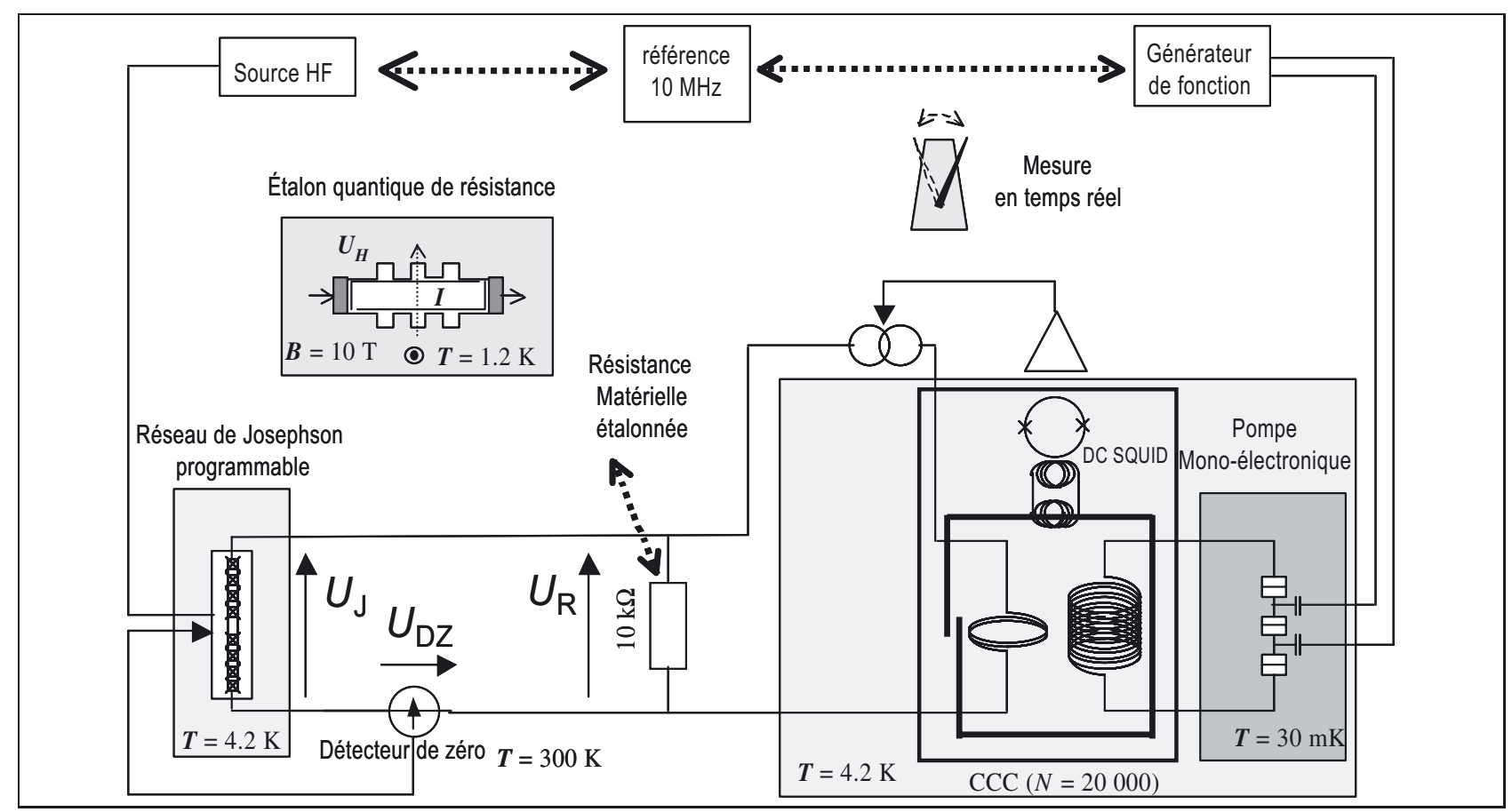

Fig. 4. - Représentation schématique du montage expérimental du «triangle métrologique » au LNE.

au regard de l'incertitude relative cible, c'est-à-dire $10^{-8}$ (excluons, pour le moment, le cas où certaines corrections se compensent). Dans le cas contraire, le triangle n'est pas fermé, un des termes de correction est significatif, mais nous ne pouvons déterminer lequel.

Par conséquent, «fermer» le triangle métrologique revient à mesurer l'écart entre le produit sans dimension $R_{\mathrm{K}} \cdot K_{\mathrm{J}} \cdot Q_{\mathrm{X}}$ et la quantité $\frac{f_{\mathrm{J}}}{f_{\mathrm{SET}}} \cdot \frac{n_{\mathrm{J}} \cdot i}{N_{\mathrm{CCC}}}$.

Le résultat de cette expérience donnera des informations sur la cohérence des trois phénomènes quantiques; et vérifier l'égalité (10) à $10^{-8}$ près (valeur de l'incertitude relative) sera un test pertinent de la validité des trois égalités (3), (4) et (6) et renforcera la confiance dans les aspects fondamentaux et universels de $K_{\mathrm{J}}$ et $R_{\mathrm{K}}$.

Obtenir une incertitude relative de $10^{-8}$, reste l'objectif principal. Atteindre cette incertitude est nécessaire pour définir les unités électriques à partir de $h$ et $e$. Cependant, des étapes intermédiaires telles que $10^{-6}$ et $10^{-7}$ peuvent s'avérer déterminantes pour, d'une part, fournir un test de quantification des futurs dispositifs mono-électroniques et d'autre part apporter des informations déjà pertinentes sur l'exactitude des relations (3) et $(6)[13,37]$. La première étape à $10^{-6}$ a déjà été récemment franchie au NIST en adoptant une autre méthode pour fermer le triangle [38]. Celle-ci s'appuie sur le chargement d'un condensateur cryogénique par comptage d'électrons au moyen d'une pompe à sept jonctions et d'un électromètre SET ; la différence de potentiel aux bornes de la capacité étant comparée à une tension Josephson. La capacité est ensuite comparée à une capacité étalon (placée à température ambiante) dont la valeur aura été préalablement étalonnée en terme de $R_{\mathrm{K}}$.

\section{Le montage expérimental}

La figure 4 décrit le montage expérimental réalisé au sein du LNE.

La pompe à électron (décrite en $§ 2.2$ ) est fixée sur l'étage de la chambre de mélange d'un réfrigérateur à dilution permettant de porter sa température à $30 \mathrm{mK}$. Le contrôle du courant électrique, électron par électron, n'est possible qu'à très basse température en minimisant les fluctuations thermiques.

Le courant quantifié généré par la pompe traverse l'enroulement primaire d'un CCC placé dans un bain d'hélium (4,2 K). Le courant est amplifié et traverse une résistance électrique matérielle étalonnée par rapport à l'étalon quantique de résistance. La valeur de cette résistance est stable et connue avec une incertitude suffisante pour espérer fermer le TMQ à $10^{-6}$ près. L'utilisation de cette résistance placée à température ambiante facilite l'expérience du triangle métrologique car la mise en œuvre de l'effet Hall quantique au sein du montage expérimental l'aurait considérablement complexifié.

Le principe de la mesure est simple : la différence de potentiel aux bornes de la résistance étalon, $U_{\mathrm{R}}$, est comparée à la tension, $U_{\mathrm{J}}$, fournie par un réseau de jonctions Josephson programmable maintenu à froid dans un cryostat indépendant rempli d'hélium liquide.

La majeure partie de l'instrumentation qui commande le dispositif SET et le réseau Josephson est pilotée par des convertisseurs numérique-analogique (DAC) afin de gérer les mesures en temps réel. 


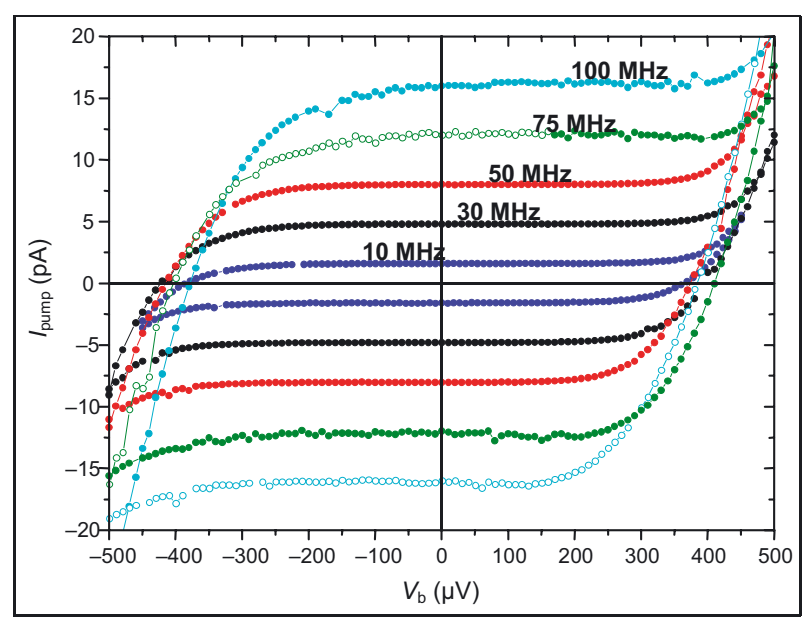

Fig. 5. - Séries de «marches de courant » pour différentes fréquences $f_{\mathrm{SET}}$ appliquées sur les électrodes de grilles (de $10 \mathrm{MHz}$ à $100 \mathrm{MHz}$ ). Le courant de pompage $I_{\text {pump }}$ est mesuré en fonction de la tension de polarisation, $V_{\mathrm{b}}$, aux bornes de la pompe.

\section{Les mesures préliminaires du courant généré par une pompe à électron}

Dans le schéma de principe de la mesure du courant généré par la pompe donné en figure 2, l'étalon quantique de courant est habituellement présenté comme le «maillon faible» de l'expérience. En effet, l'étude métrologique de la pompe à électron dans un tel contexte n'a, jusqu'à présent, jamais été réalisée. Dans un premier temps, nous avons étudié le courant maximum pouvant être généré par la pompe. Les résultats reportés ici ont tous été obtenus avec une pompe à trois jonctions fournie par la PTB dans le cadre du projet européen « COUNT ».

Un programme de simulation du diagramme de stabilité développé par Steck [33] a permis d'estimer la valeur des principaux paramètres de notre dispositif monoélectronique. Les résultats sont rassemblés dans l'article [32].

Les capacités électriques des trois jonctions notées $C_{\mathrm{L}}, C_{\mathrm{m}}, C_{\mathrm{R}}$ (respectivement gauche, milieu et droite) ont pour valeurs : $C_{\mathrm{L}}=115 \mathrm{aF}, C_{\mathrm{m}}=80 \mathrm{aF}$ et $C_{\mathrm{R}}=$ $115 \mathrm{aF}$. Les capacités des électrodes de grilles ont été estimées à $C_{\mathrm{g} 1}=35 \mathrm{aF}$ et $C_{\mathrm{g} 2}=30 \mathrm{aF}$. Un niveau de bruit blanc de $3 \mathrm{fA} / \mathrm{Hz}^{1 / 2}$ a été mesuré avec le montage expérimental complet à $1 \mathrm{~Hz}$.

La figure 5 montre des «marches de courant» dans le domaine de fréquences $f_{\text {SET }}$ de $10 \mathrm{MHz}$ à $100 \mathrm{MHz}$. Ces «marches » témoignent de la quantification du courant ( $\left.I_{\text {pump }}=e \cdot f_{\mathrm{SET}}\right)$ sur un certain domaine de tensions de polarisation aux bornes du dispositif. Pour une fréquence de $100 \mathrm{MHz}$, le courant de pompage est de 16,2 pA. Cela correspond au courant maximum que l'on peut espérer générer avec une telle pompe. Au-delà, le plateau disparaît et l'on perd la quantification. Les «marches » négatives ont été obtenues en modifiant le déphasage entre les deux tensions de grilles [32].

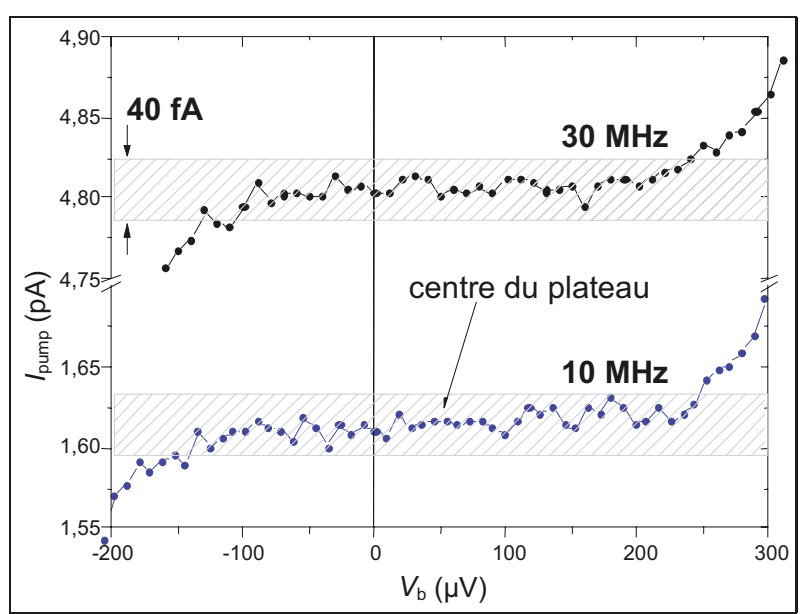

Fig. 6. - Vue grossie de deux «marches » équivalentes à celles données en figure 5 et obtenues avec une fréquence $f_{\mathrm{SET}}$ respectivement de $10 \mathrm{MHz}$ et $30 \mathrm{MHz}$.

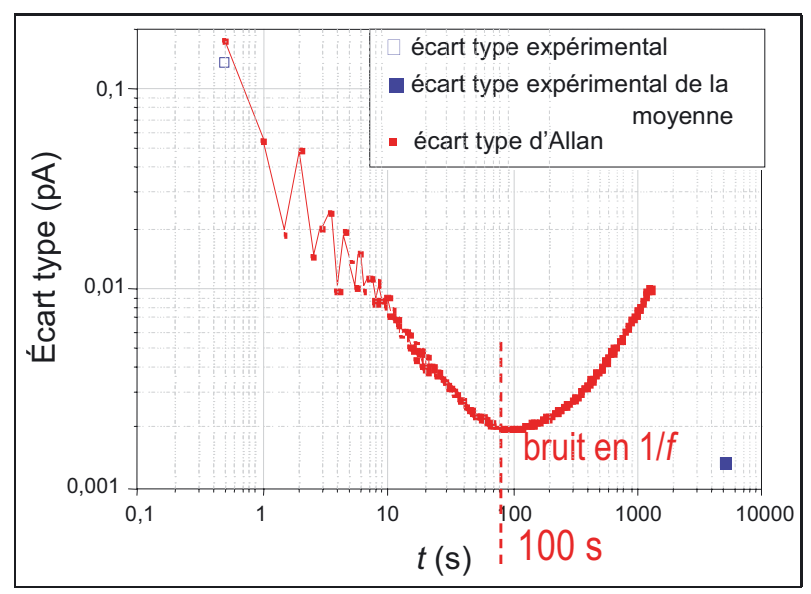

Fig. 7. - Écart type d'Allan en fonction du temps ; l'écart type expérimental et expérimental de la moyenne sont également reportés sur ce graphe.

La largeur des plateaux mesure la qualité de l'étalon de courant. Une large «marche» signifie que le courant est stable et quantifié sur un domaine important de tension de polarisation, $V_{\mathrm{b}}$. Les résultats reportés en figure 6 montre qu'une «marche » obtenue à $10 \mathrm{MHz}$ est plate sur environ $400 \mu \mathrm{V}$ si l'on considère un $\Delta I_{\text {pump }}$ de $40 \mathrm{fA}$.

L'ensemble des performances atteintes avec cette pompe à trois jonctions est, jusqu'aujourd'hui, inégalé d'après les articles publiés. Par conséquent, cette pompe de référence a été choisie pour être intégrée dans l'expérience du triangle métrologique.

Avec un dispositif mono-électronique telle que la pompe à électron, la mesure du courant est effectuée au centre du plateau. En effet, à cet endroit, les erreurs de pompage sont théoriquement minimisées. La mesure du courant généré par la pompe doit être répétée au cours du temps pour que l'incertitude soit minimale. Le calcul de l'écart type d'Allan est reporté sur la figure 7.

Cependant, l'évolution de l'écart type d'Allan en fonction du temps montre que le bruit en $1 / f$ apparaît 


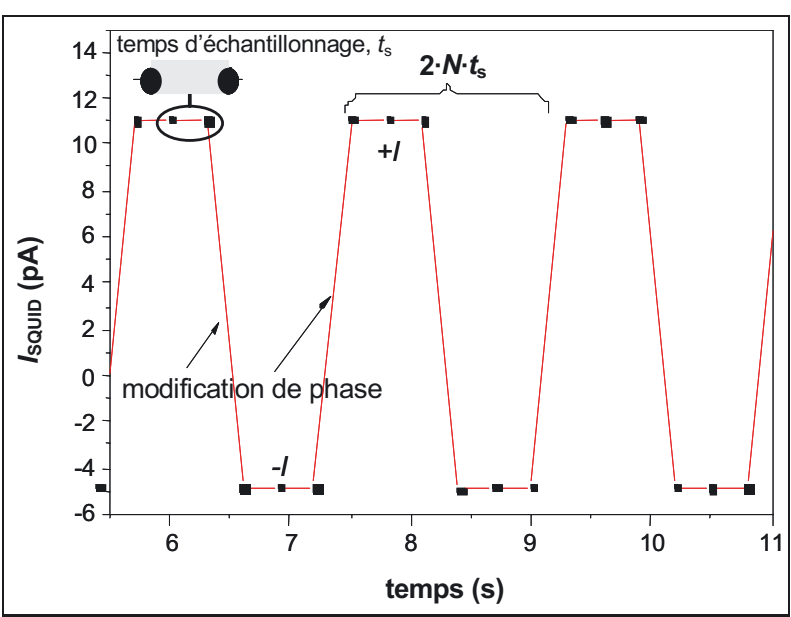

Fig. 8. - Principe de la mesure en créneaux; le sens du courant est changé périodiquement pour s'affranchir du bruit en $1 / f$ et annuler les offsets et dérive des instruments.

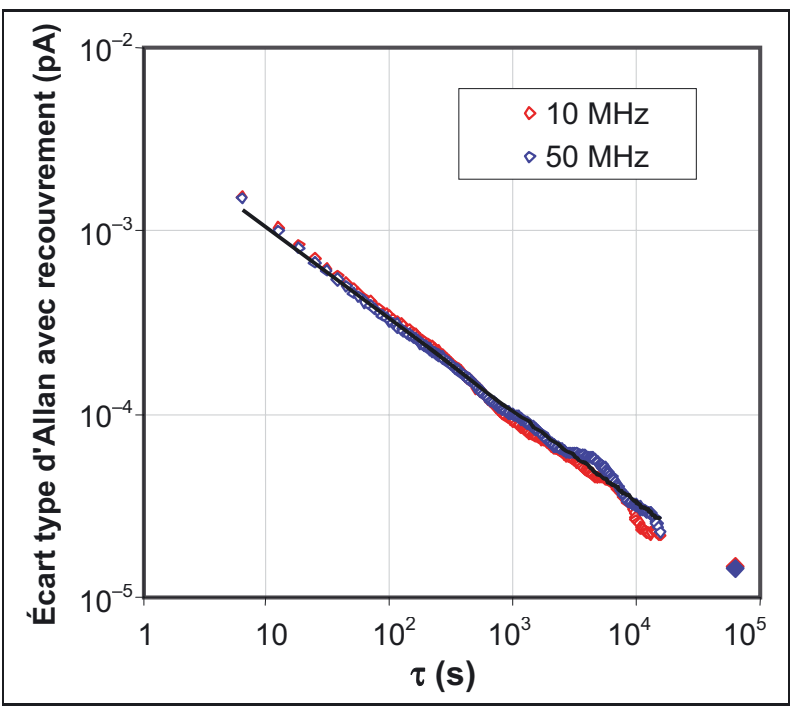

Fig. 9. - Écart type en fonction du temps d'échantillonnage. Les losanges (vides) correspondent aux calculs de l'écart type d'Allan. Les points représentent les écarts types expérimentaux de la moyenne en prenant en compte l'ensemble des données de mesures.

très tôt, après un temps de mesure de seulement $100 \mathrm{~s}$, ce qui empêche d'accumuler les données pour minimiser l'incertitude de type A. Afin de s'affranchir du bruit en $1 / f$ mais aussi des offsets et dérives du SQUID, l'état du dispositif mono-électronique est modifié en réalisant des créneaux de courant. Le principe de la mesure est schématisé en figure 8.

$N$ valeurs du courant sont mesurées pour un temps d'échantillonnage $t_{s}$ et le sens du courant est inversé après $1 \cdot N \cdot t_{s}$ secondes. Une mesure du courant correspond à la moyenne des mesures réalisées sur la période $2 \cdot N \cdot t_{s}$. Le calcul de l'écart type d'Allan est réalisé sur l'ensemble de ces moyennes et la variation en fonction du temps est donnée en figure 9 .
Cette méthode de mesure a été détaillée dans une publication précédente qui faisait état des résultats à miparcours obtenus dans le cadre de TRIMET [32]. Des résultats préliminaires avaient montré que le bruit généré par la pompe et par l'ensemble du montage était compris entre $10 \mathrm{fA} / \mathrm{Hz}^{1 / 2}$ et $15 \mathrm{fA} / \mathrm{Hz}^{1 / 2}$ pour des fréquences $f_{\mathrm{SET}}$ comprises entre $10 \mathrm{MHz}$ et $100 \mathrm{MHz}$. Une incertitude de type A de 3,9 $\times 10^{-6}$ avait été atteinte pour un courant de $16 \mathrm{pA}\left(f_{\mathrm{SET}}=100 \mathrm{MHz}\right)$.

À la fin du projet TRIMET, une modification du montage expérimental ainsi qu'un meilleur pilotage de l'instrumentation (changement des programmes LABVIEW) ont permis d'améliorer sensiblement ces résultats. Aujourd'hui, le bruit blanc est de $3 \mathrm{fA} / \mathrm{Hz}^{1 / 2}$ à $5 \mathrm{fA} / \mathrm{Hz}^{1 / 2}$. Les deux courbes d'Allan reportées en figure 9 témoignent de la stabilité de la pompe sur près de $20 \mathrm{~h}$ pour deux fréquences de pompages $\left(f_{\mathrm{SET}}=10 \mathrm{MHz}\right.$ et $50 \mathrm{MHz}$ ). L'incertitude sur la mesure du courant est de $10^{-3} \mathrm{pA}$ à $10 \mathrm{~s}$; ce qui signifie une réduction d'incertitude d'un facteur 3 à 4 par rapport aux mesures précédentes [32]. En prenant en compte l'ensemble des données, l'incertitude de type A est de $1,9 \times 10^{-6}$ en valeur relative (14 aA, Fig. 9) pour une fréquence de $50 \mathrm{MHz}$.

Ces mesures préliminaires sur la pompe à électron permettent de tirer plusieurs conclusions :

i) la mesure en créneaux a permis de s'affranchir du bruit $1 / f$, des dérives et offsets;

ii) le courant généré par la pompe est stable sur des temps de mesure pouvant atteindre $20 \mathrm{~h}$;

iii) la pompe à électron utilisée ne peut pas fournir un courant quantifié supérieur à $16 \mathrm{pA}\left(f_{\mathrm{SET}}=\right.$ $100 \mathrm{MHz})$; au-dessus de cette fréquence de pompage, les «marches de courant » disparaissent et l'intensité du courant n'est plus quantifiée ;

iv) dans la gamme de fréquences $f_{\mathrm{SET}}$ étudiée ( $10 \mathrm{MHz}-$ $100 \mathrm{MHz}$ ), le niveau de bruit blanc généré par la pompe est constant. Il est actuellement situé entre $3 \mathrm{fA} / \mathrm{Hz}^{1 / 2}$ et $4 \mathrm{fA} / \mathrm{Hz}^{1 / 2}$;

v) une incertitude relative de $1,9 \times 10^{-6}$ a été obtenue après $17 \mathrm{~h}$ de mesures pour une fréquence de pompage de $50 \mathrm{MHz}$.

\section{La mise en œuvre du triangle métrologique quantique et la mesure de $e$}

Les résultats détaillés dans la partie 4 montrent que l'incertitude relative visée de $1 \times 10^{-6}$ semble réalisable avec notre dispositif mono-électronique de référence. Le triangle métrologique a alors été mis en œuvre conformément au schéma de la figure 4 . Les commandes des différents appareils par l'intermédiaire des DAC permettent des retournements synchronisés de la tension Josephson avec le courant de la pompe à électron afin d'effectuer la mesure en créneaux. 


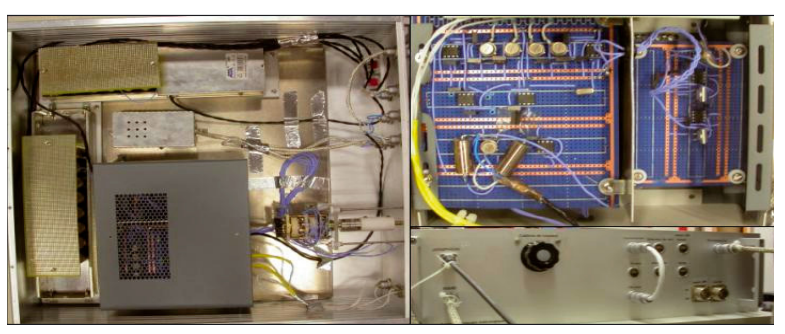

Fig. 10. - Source de courant du CCC du triangle métrologique, développée au LNE.

Le cœur de notre dispositif de mesure est l'amplificateur de courant : le CCC [36]. Il est constitué de deux enroulements de fil supraconducteur de $N_{1}$ et $N_{2}$ tours, inclus à l'intérieur d'un blindage toroïdal supraconducteur associé à un SQUID. Cet outil métrologique permet d'amplifier un courant primaire $I_{1}$ en un courant secondaire $I_{2}$ avec un rapport parfaitement connu (à $10^{-10}$ près) $N_{\text {CCC }}=N_{1} / N_{2}$. Ce système fonctionne sur la base d'une régulation du courant $I_{2}$ à la valeur $N_{\mathrm{CCC}} \cdot I_{1}$ par l'intermédiaire d'un détecteur de flux magnétique ultra-sensible, le SQUID. Le CCC est par ailleurs l'élément de base du comparateur de résistances qui permet l'étalonnage d'étalons matériels par rapport à l'étalon quantique de résistance.

Le rapport d'amplification sur le montage actuel est 20000 . En raison de l'exactitude requise pour cette expérience, il est nécessaire de pré-ajuster le rapport des courants $I_{1}$ et $I_{2}$ au rapport souhaité $N_{\mathrm{CCC}}$ par l'adjonction d'une source de courant externe au SQUID. Une source de courant très stable a été développée au laboratoire pour alimenter le comparateur (Fig. 10). Cette source, fondée sur un convertisseur tension/courant, convertit une combinaison de deux tensions de pilotage : une tension délivrée par les DAC, qui permet un pré-ajustement du courant circulant dans l'enroulement secondaire et la tension de contre-réaction générée par l'électronique du SQUID. Cette source est montée dans un coffret qui contient également des résistances étalonnées par rapport à l'effet Hall quantique, les connexions permettant de comparer la tension aux bornes de la résistance avec la tension Josephson et un amplificateur différentiel à haute impédance d'entrée de gain 110. Ce coffret offre la possibilité de connecter, en série avec l'enroulement secondaire, deux résistances internes de $1 \mathrm{k} \Omega$ et $10 \mathrm{k} \Omega$ (type Vishay VH102 K) ou bien une résistance étalon externe.

Enfin, concernant la partie «tension de référence» de l'expérience constituée par le réseau de jonctions Josephson, nous avons réalisé une source de courant programmable dédiée à cette expérience. Cette source, qui est entièrement pilotable par ordinateur (DAC), polarise chacun des quatorze segments du réseau Josephson. Elle possède également quatre sorties de tension supplémentaires qui sont utilisées pour commander la source de courant alimentant la résistance. Ce montage permet de synchroniser parfaitement le courant mono-électronique de la pompe avec celui alimentant le réseau Josephson.

Afin de vérifier le bon fonctionnement de notre montage expérimental, une série de mesures de la grandeur
Tableau 1

Paramètres de réglage de la pompe et du réseau de jonctions Josephson.

\begin{tabular}{|c|c|c|c|c|}
\hline \multicolumn{2}{|c|}{ Pompe à électron } & \multicolumn{3}{|c|}{ JAVS } \\
\hline $\begin{array}{c}f_{\text {SET }} \\
(\mathrm{MHz})\end{array}$ & $\begin{array}{c}I_{\text {pump }} \\
(\mathrm{pA})\end{array}$ & $\begin{array}{c}f_{\mathrm{J}} \\
(\mathrm{GHz})\end{array}$ & $n_{\mathrm{J}}$ & $\begin{array}{c}U_{\mathrm{J}} \\
(\mathrm{mV})\end{array}$ \\
\hline 23,55 & 3,613 & 73 & 5 & 0,754755 \\
\hline 37,69 & 6,0386 & 73 & 8 & 1,207609 \\
\hline
\end{tabular}

$\Delta e / e=\left(Q_{\mathrm{X}}-e_{\mathrm{CODATA}}\right) / e_{\mathrm{CODATA}}$ a été effectuée. Il s'agit ici de mesurer l'écart éventuel de $Q_{\mathrm{X}}$, notre estimateur de la charge, avec la valeur de la charge élémentaire publiée par Codata [39].

Nous rappelons que le principe de l'expérience du «Triangle métrologique », schématisée en figure 4, consiste à opposer la différence de potentiel, $U_{\mathrm{R}}$, aux bornes de la résistance étalonnée traversée par le courant mono-électronique amplifié, $N_{\mathrm{CCC}} \cdot I_{\text {pump }}$, avec la tension de référence Josephson, $U_{\mathrm{J}}$. Par conséquent, la grandeur mesurée est la tension de déséquilibre de ce pont, $U_{\mathrm{DZ}}$, au moyen du détecteur de «zéro » (nanovoltmètre $3458 \mathrm{~A}$ ). Nous avons alors l'égalité (16).

$$
U_{\mathrm{J}}=U_{\mathrm{DZ}}+U_{\mathrm{R}}
$$

Dans cette partie, toutes les mesures ont été réalisées avec la résistance, étalonnée par rapport à l'effet Hall quantique, de valeur : $R=10000 \Omega$.

D'après les expressions (1) et (7), nous tirons de la relation (16) :

$$
Q_{\mathrm{X}}=\frac{\frac{n_{\mathrm{J}} \cdot f_{\mathrm{J}}}{K_{\mathrm{J}}}-U_{\mathrm{DZ}}}{R \cdot N_{\mathrm{CCC}} \cdot f_{\mathrm{SET}}} .
$$

Nous obtenons ainsi une mesure de l'estimateur de la charge de l'électron. Dans cette première étape de fermeture du triangle métrologique, notre objectif est d'atteindre une incertitude relative de $10^{-6}$. Par conséquent, avec cette valeur, nous n'avons aucun doute sur la valeur de $K_{\mathrm{J}}$, l'égalité (3) étant vérifiée.

La stabilité de la tension de déséquilibre, $U_{D Z}$, est mesurée grâce à l'évolution temporelle de l'écart type d'Allan présentée en figure 11. La pompe a été cadencée à deux fréquences différentes de pompage $\left(f_{\mathrm{SET}}\right)$ : 23,55 MHz et 37,69 MHz. Le réseau Josephson étant soumis à un rayonnement électromagnétique de fréquence $73 \mathrm{GHz}$, cinq et huit jonctions ont été polarisées afin que la tension Josephson corresponde à ces deux fréquences de pompage. L'ensemble de ces paramètres est rassemblé dans le tableau 1.

L'évolution linéaire de la représentation « $\log -\log$ » de l'écart type d'Allan au cours du temps révèle que le bruit est «blanc» sur la totalité du temps de mesure. En outre, l'amplitude du bruit en tension correspond à une amplitude de bruit en courant de l'ordre de $8 \mathrm{fA} / \mathrm{Hz}^{1 / 2}$ à $10 \mathrm{fA} / \mathrm{Hz}^{1 / 2}$. Cette amplitude est supérieure à celle annoncée en partie 4 (Fig. 9). Il est à noter que les mesures 


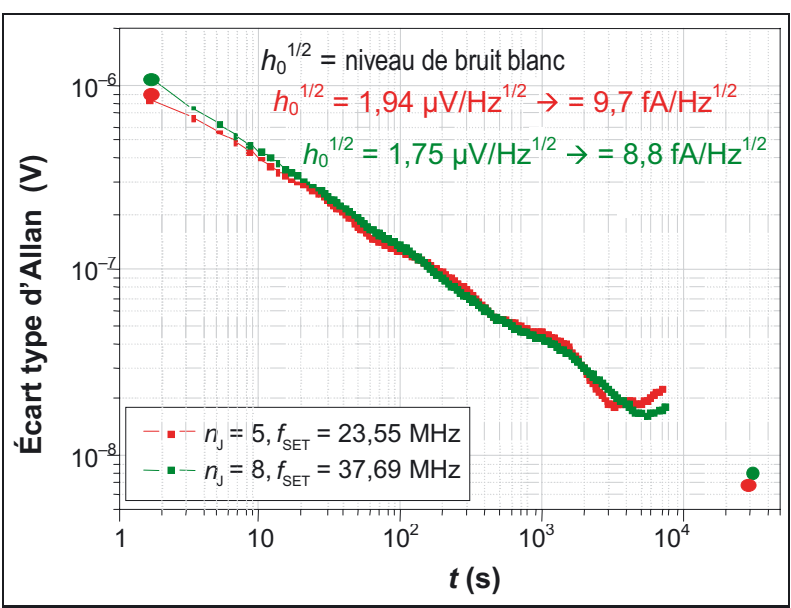

Fig. 11. - Écart type des mesures faites avec le détecteur de « zéro », $V_{D Z}$, en fonction du temps d'échantillonnage; les courbes correspondent aux calculs de l'écart type d'Allan; les points (en bas à droite) représentent les écarts types expérimentaux de la moyenne en prenant en compte l'ensemble des résultats de mesure.

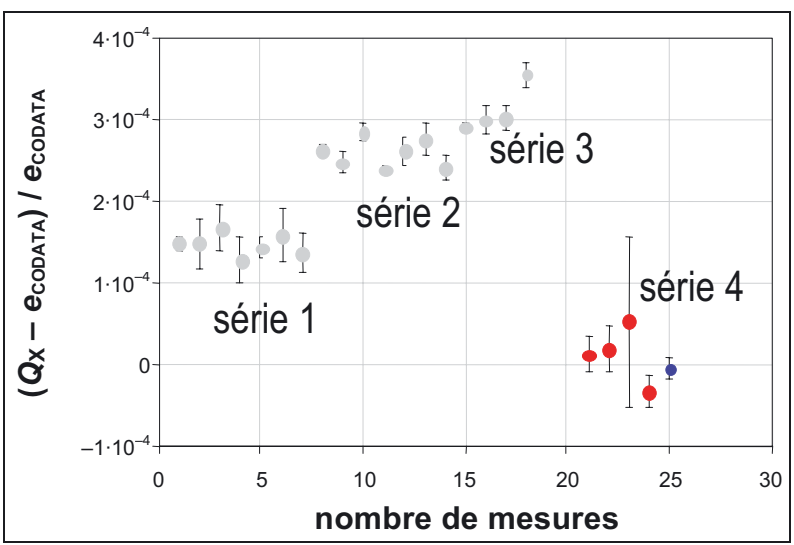

Fig. 12. - Différentes séries de mesure de l'écart de $Q_{\mathrm{X}}$ à la valeur CODATA de $e$ avec le montage du TMQ. Les trois premières séries ont été obtenues pendant la durée du projet et la quatrième série a été réalisée durant les mois suivants la fin du projet. Le dernier point de cette quatrième série correspond à la moyenne des points de la série.

ont été effectuées après les modifications détaillées dans la partie 4 .

À la fin du projet, des séries de mesures de $\left(Q_{\mathrm{X}}-\right.$ $\left.e_{\text {CODATA }}\right) / e_{\text {CODATA }}$ ont été effectuées et sont reportées en figure 12.

Les séries 1 à 3 ont été effectuées au cours du projet TRIMET. Les points de la série 1 montrent un écart relatif d'environ $1,5 \times 10^{-4}$ entre $Q_{\mathrm{x}}$ et la valeur recommandée par Codata pour la charge élémentaire. Après modification de l'état du montage expérimental (certains appareils sont éteints puis rallumés, le réseau Josephson subit un cyclage thermique...), l'écart se creuse (série 3 et 4). Deux conclusions s'imposent :

1) un écart systématique apparaît au cours d'une série de mesures $\left(\sim 1,4 \times 10^{-4}\right.$ sur la série 1$)$. Cet écart semble reproductible au sein d'une même série;
2) en revanche, cet écart devient non reproductible d'une série à l'autre.

Ce manque de reproductibilité a été imputé à un problème lié à l'existence de plusieurs points de mise à la masse dans le montage expérimental.

À l'issue du projet, les deux points de mise à la masse ont été découplés au moyen d'amplificateurs d'isolement. Les programmes de pilotage (Labview) ont été révisés et améliorés, comme évoqué dans la partie 4 de l'article.

Une nouvelle série de mesure (série 4), avec un écart de $Q_{\mathrm{x}}$ à la valeur CODATA de $e$ inférieur à $5 \times 10^{-5}$ (Fig. 12) a été faite en juin 2009. Les problèmes de défaut de reproductibilité semblent avoir disparus. Des mesures complémentaires sont cependant nécessaires avant de conclure.

À partir de quatre points, une valeur moyenne pondérée égale à $Q_{\mathrm{X}}=1,602168 \mathrm{C}$ (écart inférieur à $-5 \times 10^{-6}$ avec la valeur CODATA) entachée d'une incertitude relative de $1,3 \times 10^{-5}$ a été déterminée.

\section{Conclusion et perspectives}

Avant d'atteindre l'objectif final de $10^{-8}$ et afin de prouver la pertinence du montage expérimental réalisé, nous avons choisi, dans le cadre du projet TRIMET, de fermer le triangle à $10^{-6}$ près. À ce niveau d'incertitude, aucun doute n'existe sur les théories décrivant les effets Hall quantique et Josephson. Cette étape intermédiaire est une opportunité pour une caractérisation métrologique de l'étalon de courant du LNE. Un éventuel écart à la quantification supérieur à $10^{-6}$ ainsi qu'une dépendance en fréquence de cet écart peuvent être mis en évidence grâce à ce nouveau montage.

L'ensemble des résultats obtenus au cours et à la suite de ce projet montre qu'une fermeture du triangle métrologique quantique à $1 \times 10^{-6}$ près, en empruntant la méthode de la loi d'Ohm semble très proche. Nous avons montré tout d'abord que la pompe pouvait générer un courant stable sur des durées de mesures supérieure à $20 \mathrm{~h}$. Une incertitude relative de $1,9 \times 10^{-6}$ a été obtenue en $17 \mathrm{~h}$. De plus, une série de mesures récentes montre un écart à la quantification du courant généré par le dispositif monoélectronique inférieur à $5 \times 10^{-6}$ pour une fréquence de pompage de $22,65 \mathrm{MHz}\left(I_{\mathrm{pump}}=3,6289 \mathrm{pA}\right)$. Ces résultats doivent toutefois encore être reproduits.

Cependant, l'objectif intermédiaire de $10^{-7}$ semble difficile à atteindre avec les pompes à électron métalliques utilisées au laboratoire. Le courant limite d'environ 16,2 pA délivré par celles-ci est un handicap certain pour tenter d'améliorer l'incertitude de mesure. Des dispositifs alternatifs capables de générer un courant supérieur, proche de $1 \mathrm{nA}$, sont actuellement proposés par les physiciens et sont en cours de test dans différents instituts de métrologie. Parmi les plus prometteurs, trois candidats possibles sont présentés en figure 13.

Le développement du nanofil quantique de GaAs/ AlGaAs est le fruit d'une collaboration entre l'université 


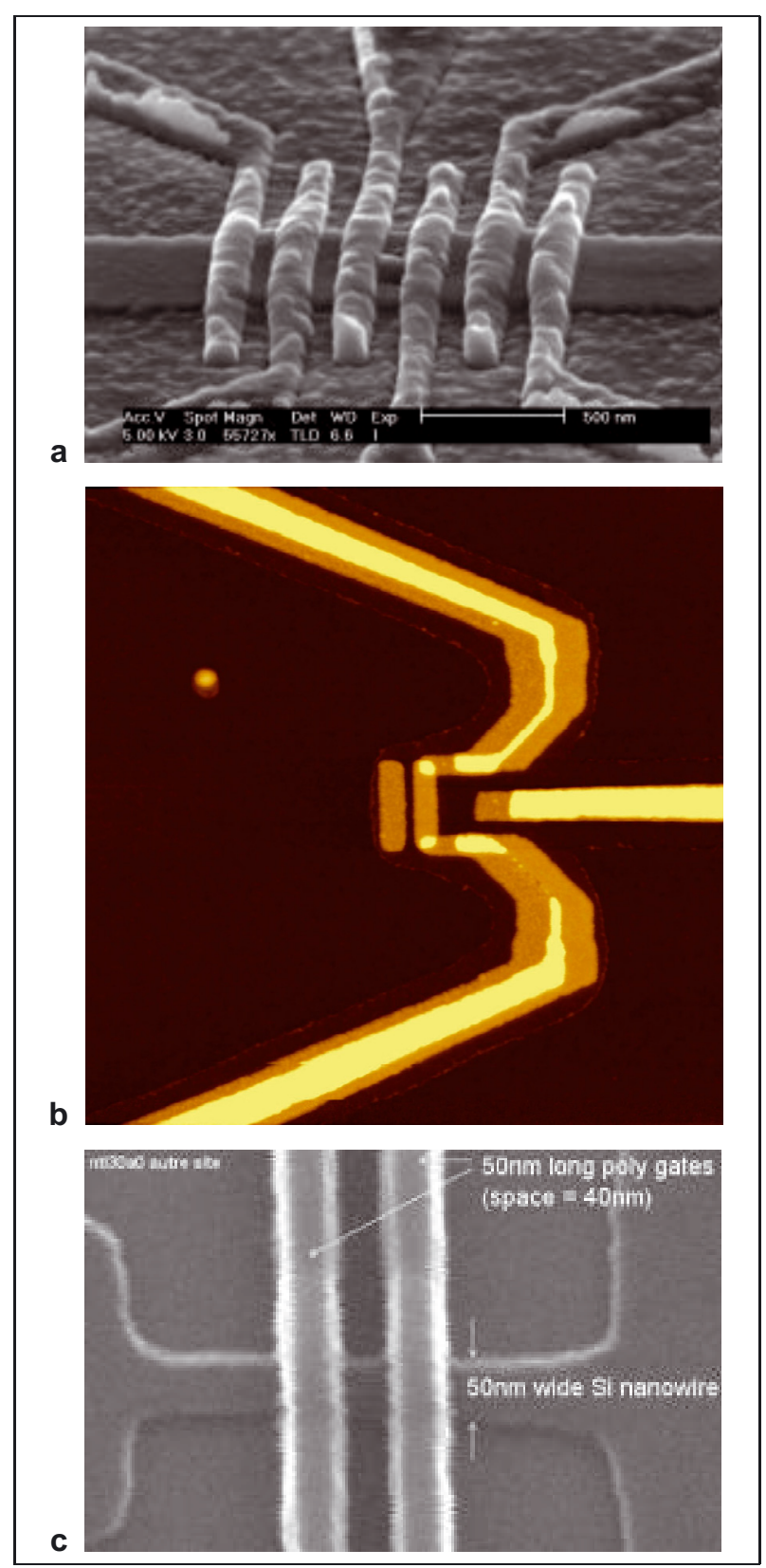

Fig. 13. - (a) Nanofil quantique d'hétérostructure GaAs/ AlGaAs [40]. (b) Tourniquet à électron hybride métalsupraconducteur [41]. (c) Nanofil de silicium [42].

de Cambridge, le NPL et la PTB. Le mécanisme de pompage diffère de celui de la pompe métallique. Dans un dispositif classique à blocage de Coulomb, le transport est fondé sur la traversée d'une barrière de potentiel par effet tunnel et dans ce cas, le caractère ondulatoire de l'électron est pris en compte. Dans ce nanofil quantique, les électrons sont considérés comme des particules qui flottent sur une vague créée par des lignes déposées sur le nanofil (Fig. 13a). Ces lignes métalliques font office de grilles et viennent modifier le potentiel local en différents points. Le blocage de Coulomb assure la quantification des électrons piégés au creux de la vague. Ici, le nombre d'électrons transportés de la source vers le drain à chaque cycle est modulable, l'intensité du courant s'écrit : $I=n \cdot e \cdot f$. Ce dispositif a montré qu'il était capable de générer un courant cadencé jusqu'à $3,4 \mathrm{GHz}$ avec une incertitude de pompage de $10^{-4}$.

Le second dispositif (Fig. 13b) ressemble à un transistor SET mais dont l'îlot et le circuit source/drain sont de nature différente. L'îlot est supraconducteur avec un circuit normal, ou le contraire. Il n'est piloté que par une unique grille donc il ouvre la voie à une mise en parallèle de dispositifs mono-électroniques et fonctionne comme un tourniquet à électron. Des plateaux de courant sont observés aux multiples de « $e \cdot f »$, lorsque l'amplitude du signal appliqué à la grille augmente. Pour le moment, l'intensité maximum du courant est faible (20 pA) et la stabilité du dispositif est médiocre mais les possibilités d'amélioration sont nombreuses.

Les propriétés métrologiques de ces deux types de dispositifs seront prochainement testées au LNE dans le cadre d'un projet européen (REUNIAM).

Un troisième candidat possible pour l'élaboration d'un étalon quantique de courant est la pompe à silicium. Le fonctionnement est complètement similaire à celui de la pompe en aluminium mais la technologie silicium permet de bénéficier des avantages de l'environnement de la microélectronique. De plus cette pompe peut être cadencée à des fréquences qui théoriquement peuvent dépasser $1 \mathrm{GHz}$ et elle est moins sensible aux charges de substrat donc plus stable. Le développement des nanofils en silicium est réalisé dans le cadre d'un projet ANR (POESI) auquel le LNE participe et dont la tâche est d'étudier les caractéristiques métrologiques de ces systèmes.

En outre, parallèlement à cette recherche du dispositif mono-électronique idéal, une seconde voie d'amélioration du montage du «triangle métrologique » est la mise au point de nouveaux CCC. L'utilisation de SQUID plus sensibles et générant moins de bruit, l'augmentation du nombre de tours de l'enroulement primaire ou l'adoption d'une configuration géométrique réduisant l'influence du champ magnétique résiduel, peuvent contribuer à diminuer de manière notable l'incertitude finale.

Enfin, l'association d'un dispositif monoélectronique à un CCC suscite l'espoir à terme d'une redéfinition «quantique » de l'ampère dont la définition actuelle est inadaptée à la physique moderne. Un étalon de courant mono-électronique permettrait une définition « naturelle » de l'ampère comme le produit d'une charge et d'une fréquence et serait bien sûr compatible avec un nouveau système SI fondé sur des constantes fondamentales.

\section{Références}

[1] BordÉ Ch.J., "Fundamental metrology/Métrologie fondamentale", C.R. de l'Académie des sciences, Physique, 5, 2004.

[2] QuinN T.J. et BURnETT K., "Royal Society Discussion Meeting: the fundamental constants of physics, precision measurements and the base units of the SI", Phil. Trans. R. Soc. Lond., A 363, 2005, 2097-327. 
[3] Piquemal F. et Jeckelmann B., "Quantum metrology and fundamental constants", Eur. Phys. J. Special Topics, 172, 2009.

[4] «Le Système international d'unités », BIPM, $8^{\mathrm{e}}$ édition, 2006.

[5] «Étapes préalables à de nouvelles définitions du kilogramme, de l'ampère, du kelvin et de la mole en fonction de constantes fondamentales », Recom-mandation 1 (CI2005) du CIPM.

[6] Eichenberger A., Genevès G. et Gournay P., "Determination of the Planck constant by means of a watt balance", Eur. Phys. J. Special Topics, 172, 2009, 363383.

[7] Pitre L., Guianvarc'h C., Sparasci F., Guillou A., Truong D., Hermier Y. et Himbert M.E., "An improved acoustic method for the determination of the Boltzmann constant at LNE-INM/CNAM", C.R. de l'Académie des sciences, Physique, 10, 2009, 835-848.

[8] Djerroud K., Lemarchand C., Gauguet A., Daussy C., BRiaudeau S., Darquié B., Lopez O., AMY-KLeIN A., Chardonnet C. et BordÉ C.J., "Measurement of the Boltzmann constant by the Doppler broadening technique at a $3.8 \times 10^{-5}$ accuracy level", C.R. de l'Académie des sciences, Physique, 10, 2009, 883-893.Un

[9] Piquemal F., Bounouh A., Devoille L., Feltin N., THEVENOT O. et TRAPON O., "Fundamental electrical standards and the quantum metrological triangle", C.R. de l'Académie des sciences, Physique, 5, 2004, 857-879.

[10] Feltin N., Devoille L., Steck B., Piquemal F., ULYSSE C. et JIN Y., «Un nouvel outil pour la métrologie électrique : le dispositif à un électron », Revue française de métrologie, 2, 2005, 11-34.

[11] LiKharev K. et Zorin A., "Theory of the Bloch-wave oscillations in small Josephson junctions", J. Low Temp. Phys., 59, 1985, 347-382.

[12] PiQuemal F. et Genevès G., "Argument for a direct realization of the quantum metrological triangle", Metrologia, 37, 2000, 207-211

[13] Keller M.W., "Current status of the quantum metrology triangle", Metrologia, 45, 2008, 102-109.

[14] JosephSON B.D., "Possible new effects in superconductive tunnelling", Phys. Lett. 1, 1962, 251.

[15] Klitzing K., Dorda G. et PePPER M., "New method for high-accuracy determination of the fine structure constant based on quantum Hall resistance", Phys. Rev. Lett., 45, 1980, 494.

[16] Clarke J., "Experimental comparison of the Josephson voltage-frequency relation in different superconductors", Phys. Rev. Lett., 21, 1968, 1566-1568.

[17] Tsai J.S., JaIN A.K. et LuKENS J.E., "High-precision test of the universality of the Josephson voltage-frequency relation", Phys. Rev. Lett., 51, 1983, 316.

[18] JAIN A.K., LUKENS J.E. et TSAI J.S., "Test for relativistic gravitational effects on charged particles", Phys. Rev. Lett. 58, 1987,1165
[19] Hartland A., Jones K., Williams J.M., GallagheR B.L. et GALlOWAY T., "Direct comparison of the quantized Hall resistance in gallium arsenide and silicon", Phys. Rev. Lett. 66, 1991, 969-973.

[20] JeckelmanN B., Inglis A.D. et JeAnNERET B., "Material, device, and step independence of the quantized Hall resistance", IEEE T. Instrum. Meas. 44, 1995, 269272.

[21] JeCKelmann B. et JeAnNERET B., "The quantum Hall effect as an electrical resistance standard", Rep. Prog. Phys., 64, 2000, 1603-1655.

[22] SCHOPFER F. et PoIRIER W., "Testing universality of the quantum Hall effect by means of the Wheatstone bridge", J. Appl. Phys., 102, 2007, 054903.

[23] SCHOPFER F. et POIRIER W., "Reproducibility of the quantum Hall effect in GaAs/AlGaAs two dimensional electron gas", Proc. of the Conference on Precision Electromagnetic Measurements (CPEM), ed. A.H. Cookson and T. Winter, 22, Boulder, 2008.

[24] Novoselov K.S., Geim A.K., Morozov S.V., JiAnG D., KATSNELSON M.I., GRIGORIEVA I.V., DUbONIS S.V. et FIRSOV A.A., "Two-dimensional gas of massless Dirac fermions in graphene", Nature, 438, 2005, 197.

[25] Tzalenchuk A., Lara-Avila S., Kalaboukhov A., PAOLILlo S., SYVÄJÄRVI M., YAKIMOVA R., KAZAKOVA O., JansSen T.J.B.M., FAL'Ko V. et KubatKin S., "Towards a quantum resistance standard based on epitaxial graphene", Nature Nano., 5, 2010, 186-189.

[26] POIRIER W. et SCHOPFER F., "Can graphene set new standards ?", Nature Nano., 5, 2010, 171-172.

[27] PIQUEMAL F., «Étalons électriques fondamentaux passifs », Revue des Techniques de l'Ingénieur, R905 et Doc $R 905,2003$.

[28] JEANNERET B. et BENZ S., "Application of the Josephson effect in electrical metrology", Eur. Phys. J. Special Topics, 172, 2009, 181-206.

[29] POIRIER W. et SCHOPFER F., "Resistance metrology based on the quantum Hall effect", Eur. Phys. J. Special Topics, 172, 2009, 207-245.

[30] Fulton T.A. et Dolan G.J., "Observation of singleelectron charging effects in small tunnel junctions", Phys. Rev. Lett., 59, 1987, 109-112.

[31] LikHAREV K.K., "Single Electron Devices and Their Applications", Proc. IEEE, 87, 1999, 606-632.

[32] Steck B., Gonzalez-Cano A., Feltin N., Devoille L., Piquemal F., Lotkhov S. et Zorin A.B., "Characterization and metrological investigation of an R-pump with driving frequencies up to $100 \mathrm{MHz}$ ", Metrologia, 45, 2008, 482-491. Un

[33] Steck B., «Application en métrologie électrique de dispositifs monoélectroniques : vers une fermeture du triangle métrologique », Thèse de doctorat de l'Université de Caen, Electronique et micro-électronique, 28 novembre 2007.

[34] Zorin A.B., LOTKHOV S.V., ZANGERLE H. et NIEMEYER J., "Coulomb blockade and cotunneling in single electron circuits with on-chip resistors : Towards the implementation of the R pump", J. Appl. Phys., 88, 2000, 2665. 
[35] HARVEY I.K., "A precise low temperature dc ratio transformer", Rev. Sci. Instrum., 43, 1972, 1626-1629.

[36] Piquemal F. et Gallop J., «Une revue des applications métrologiques des SQUID », Revue française de métrologie, $\mathbf{8}, 2006,13-43$.

[37] Feltin N. et PiQuemal F., "Determination of the elementary charge and the quantum metrological experiment", Eur. Phys. J. Special Topics, 172, 2009, 267-296.

[38] Keller M.W., Zimmerman N.M. et EicheNBerger A.L, "Uncertainty budget for the NIST electron counting capacitance standard, ECCS-1", Metrologia, 44, 2007, 505-512.

[39] Mohr P.J., TAYlor B. N. et Newell D., "CODATA recommended values of the fundamental physical constants : 2006", Rev. Modern Phys., 80, 2008, 633-730.
[40] Blumenthal M.D., Kaestner B., Li L., Giblin S., JANSSEN T.J.B.M., PEPPER M., ANDERSON D., JONES G. et RITCHIE D.A., "Gigahertz quantized charge pumping", Nature Phys., 3, 2007, 343-347.

[41] Pekola J.P., Vartiainen J.J., Mottonen M., Saira OP., MeschKe M. et AVERIN D.V., "Hybrid single-electron transistor as a source of quantized electric current", Nature Phys., 4, 2008, 120-124.

[42] Pierre M., Jehl X., SANQuer M., Vinet M., Previtali B., Molas G. et Deleonibus S., "Low noise silicon CMOS single-electron transistors and electron pumps", Proc. of the Conference on Precision Electromagnetic Measurements (CPEM), ed. A.H. Cookson and T. Winter, 282, Boulder, 2008.

Article reçu le 21 janvier 2010, version révisée reçue le 7 juin 2011. 